\title{
COVID-19 and cancer care: what do international guidelines say?
}

\author{
Alfredo Tartarone ${ }^{1}\left[\right.$ [ $\cdot$ Rosa Lerose $^{2}$ \\ Received: 12 May 2020 / Accepted: 29 July 2020 / Published online: 7 August 2020 \\ (c) Springer Science+Business Media, LLC, part of Springer Nature 2020
}

\begin{abstract}
Cancer patients are at particular risk from COVID-19 since they usually present multiple risk factors for this infection such as older age, immunosuppressed state, comorbidities (e.g., chronic lung disease, diabetes, cardiovascular diseases), need of frequent hospital admissions and visits. Therefore, in the COVID era, oncologists should carefully weigh risks/benefits when planning cancer therapies and follow-up appointments. Recently, several scientific associations developed specific guidelines or recommendations to help physicians in their clinical practice. This review focuses on main available guidelines/ recommendations regarding the cancer patient management during the COVID-19 pandemic.
\end{abstract}

Keywords COVID-19 Pandemic · Guidelines $\cdot$ Cancer patients

\section{Introduction}

On March 11 2020, the World Health Organization (WHO) declared the worldwide outbreak of the novel coronavirus disease 2019 (COVID-19) to be a pandemic [1]. The rapid spread of the COVID-19 epidemic opened unprecedented challenges throughout the world. It is known that the virus can spread via respiratory droplets and direct contact, as well as potentially through airborne routes of spread [2]. Current WHO recommendations emphasized the importance not only of an appropriate use of all personal protective equipment (PPE) from health care workers, but also the need for all people of frequent hand hygiene, environmental cleaning and disinfection, as well as the usefulness of maintaining physical distances and avoidance of close, unprotected contact with persons with fever or respiratory symptoms [2].

Cancer may be a risk factor in case of COVID-19 infection. In fact, it has been demonstrated that the risk of COVID-19 increases with age and the presence of comorbidities, including cancer [3]. Consequently cancer patients

Alfredo Tartarone

tarta1@ virgilio.it

1 Division of Medical Oncology, Department of Onco-Hematology, IRCCS-CROB Referral Cancer Center of Basilicata, Via Padre Pio 1, 85028 Rionero in Vulture (PZ), Italy

2 Hospital Pharmacy, IRCCS-CROB Referral Cancer Center of Basilicata, Rionero in Vulture (PZ), Italy are at very high risk for COVID-19 infection considering that they often present multiple risk factors as well as older age, immunosuppressed state due to anticancer treatment and several comorbidities (e.g., chronic lung disease, diabetes, cardiovascular diseases). In addition, as it is known, they need frequent hospital admissions and visits that represent a recognized potential risk factors for COVID-19 infection. Recent evidences from China indicated that cancer patients, especially lung cancer patients, might have more severe symptoms and poorer outcomes from COVID-19 infection than persons without cancer [4-6]. In addition, the report from the Italian national medical council, regarding the characteristics of patients who have died from COVID-19 in Italy, showed the presence of $16.3 \%$ of cancer patients [7]. Also a recent study published by researchers of the Albert Einstein College of Medicine and Montefiore Health System (New York) confirmed that the mortality rate among cancer patients infected with COVID-19 is higher [8].

For all these reasons, the current emergency appears to be of particular concern to the oncologists and their patients; more specifically, in the COVID era, oncologists should carefully weigh risks/benefits when planning cancer therapies and follow-up appointments. Recently, several scientific associations developed peculiar guidelines or recommendations to help physicians in their clinical practice.

This review focuses on main available guidelines/recommendations regarding the cancer patient management during the COVID-19 pandemic. 


\section{The AIOM (Italian Association of Medical Oncology) recommendations}

The recently published Italian recommendations advise to evaluate case by case the possibility of postponing a treatment, considering the biological aspects of cancer, the clinical characteristics of the patient and the potential health risks for COVID-19 infection [9]. With regard to the followup visits, it is considered appropriate to postpone them for a few months after a telemedicine/phone contact, confirming only the visits that cannot be delayed due to clinical needs. The admission to an oncology unit is forbidden in case of fever and/or respiratory symptoms, without having performed a medical triage. Home patients who develop these symptoms should call their healthcare provider first. AIOM also recommends limiting access into the hospital for patient accompanists to avoid overcrowding in waiting rooms and in areas where chemotherapy is carried out. The use of surgical masks is recommended for all healthcare professionals, patients and their accompanists; healthcare professionals should wear gloves and FFP2-FFP3 masks, if available.

On April 25 2020, AIOM also published guidelines about the management of breast cancer patients during COVID-19 pandemic [10].

\section{The French guidelines}

According to French guidelines, clinical management of cancer patients during the COVID-19 pandemic should be based on the following priority order: curative or non-curative intent of the treatment, age, life expectancy, time since diagnosis and symptoms [11]. Every effort should be made to keep medical and radiation oncology units "COVID-19 sanctuaries"; therefore, cancer patients positive for COVID19 should be directed to specialized COVID units and not into oncology departments. In addition, several measures such as the following ones should be encouraged to reduce the presence of cancer patients in the hospital: telemedicine/phone calls, replacement of intravenous drugs with oral drugs, home administration of subcutaneous anticancer agents and adjustment of dosing schedules of chemotherapy or radiotherapy. To protect cancer patients separation methods should be adopted as well as maintaining a minimum space between seats or wearing of masks.

\section{The NICE (National Institute for Health and Care Excellence) guidelines}

The NICE guidelines include a series of measures to minimize face-to-face contact, e.g., offering telephone or telemedicine consultations (in particular for follow-up visits), using home delivery services for medicines or local services for blood tests [12]. Moreover, if possible, patients should attend appointments without family members or carers to reduce the risk of contracting or spreading the infection. To establish the intent of the anticancer treatment, NICE introduced a priority scale with six levels Table 1 . The level one includes curative treatments with a high (more than 50\%) chance of success, while level 6 is reserved for non-curative therapies with an intermediate (15\% to 50\%) chance of palliation and less than 1 year expected extension of life. Prioritization decisions should be made by a multidisciplinary team and should be clearly communicated to patients and their families also with written documentation, if possible.

If a patient has COVID-19 infection NICE suggests to continue systemic anticancer treatment only if it is necessary for an urgent control of the disease, otherwise it is advisable to postpone the treatment until the patient has at least one negative test for COVID-19. All healthcare workers involved in caring for patients who have known or suspected COVID19 should use PPE.

\section{The ESMO (European Society of Medical Oncology) recommendations}

First of all, ESMO identified the following categories of patients as potentially at risk for COVID-19: patients receiving chemotherapy or who have received chemotherapy in the last 3 months; patients receiving extensive

Table 1 NICE priority scale for systemic anticancer treatment [12]

\begin{tabular}{|c|c|}
\hline Priority level & Treatment intent and risk/benefit ratio \\
\hline 1 & Curative treatment with a high (more than $50 \%$ ) chance of success \\
\hline 2 & Curative treatment with an intermediate ( $15 \%$ to $50 \%$ ) chance of success \\
\hline 3 & Non-curative treatment with a high (more than $50 \%$ ) chance of more than 1 year extension to life \\
\hline 4 & $\begin{array}{l}\text { Curative therapy with a low ( } 0 \% \text { to } 15 \%) \text { chance of success or non-curative therapy with an intermediate (15\% to } 50 \%) \text { chance of } \\
\text { more than } 1 \text { year extension to life }\end{array}$ \\
\hline 5 & $\begin{array}{l}\text { Non-curative therapy with a high (more than } 50 \% \text { ) chance of palliation or temporary tumor control and less than } 1 \text { year expected } \\
\text { extension to life }\end{array}$ \\
\hline 6 & $\begin{array}{l}\text { Non-curative therapy with an intermediate ( } 15 \% \text { to } 50 \%) \text { chance of palliation or temporary tumor control and less than } 1 \text { year } \\
\text { expected extension to life }\end{array}$ \\
\hline
\end{tabular}


radiotherapy; patients who have undergone bone marrow or peripheral blood stem cell transplantation in the last 6 months; patients with some types of hematological malignancies; specific group of cancer patients with an impaired immune system [13]. Patients can be also divided in two groups: patients off therapy (A) and patients under treatment (B). For all patients (A and B) it is mandatory to provide health education such as hand hygiene, maintaining physical distances, avoid unprotected contact with persons with fever or respiratory symptoms and wear PPE during hospital admissions or visits. Regarding patients receiving active treatment (B) physicians should discuss with them the benefits and risks of the therapy, identify specific pathways to guarantee the timing of the treatment (e.g., prioritize adjuvant therapies in patients with highrisk disease), modify regimen schedules to reduce number of clinic visits (e.g., three or two weekly as opposed to weekly, oral or subcutaneous vs IV), favor phone or webtechnology contacts.

In addition, ESMO established a tiered approach for categorizing patients into three levels of priority (high, medium and low) to receive active cancer treatment during the COVID-19 pandemic Table 2. High priority identifies a life threatening condition that requires a rapid intervention, low priority conversely, is reserved for patients with a stable clinical condition that allows to delay further interventions for the duration of the COVID-19 pandemic. According to the tiered approach, ESMO developed the socalled "COVID-19 era recommendations" for the management of the main types of tumors. ESMO also suggested that Cancer Hubs should be provided of checkpoint areas for early detection of potentially infectious persons and that RT-PCR SRAS-CoV-2 testing should be proposed to all patients undergoing active treatment, ideally before each cycle; if not feasible, it should be considered at least in patients with suspected COVID-19 infection. In the same way serology, if feasible, could be evaluated in all cancer patients to identify previous COVID-19 infection.

Finally, ESMO has launched an international project, the ESMO-CoCARE Registry, to quickly gather data from real world about cancer patients with suspected or confirmed COVID-19.

\section{The ASCO (American Society of Clinical Oncology) recommendations}

The ASCO website reports a great deal of information on patient care and COVID-19, along with links to guidance from organizations such as the US Centers for Disease Control and Prevention (CDC) [14, 15]. All patients should be informed on COVID-19 associated symptoms and instructed on appropriate hand hygiene. It is essential to limit hospital access, establish triage stations outside the clinic, convert waiting areas and infusion suites to allow at least six feet distance between patients. Physicians should postpone follow-up visits of patients who are not on active cancer treatment and provide patient communication via telemedicine/phone calls. Home collection of lab samples may be considered as well as the evaluation of laboratory test results can be performed by the health care team and communicated to the patients. ASCO emphasized the risk of drug shortages caused by drug production impairment due to COVID-19 outbreaks and invited its member to a judicious use of any scarce resources. However, U.S. FDA (Food and Drug Administration) maintains a list of drugs in shortage and is working with manufacturers to ensure a constant supply. ASCO is also aware that conducting clinical trials will be particularly difficult during this time. EMA (European Medicines Agency), U.S. FDA and U.S. NCI (National Cancer Institute), anyhow, have just issued guides on managing clinical trials during COVID-19 pandemic [16-18]. For a series of other general recommendations ASCO suggests to refer to CDC guidance for COVID-19 [15].

\section{Conclusion}

It is well established that cancer patients are at higher risk of COVID-19 infection and that cancer is an independent risk factor for adverse outcome and mortality from COVID-19.

The last ASCO annual meeting hosted a special session on cancer and COVID-19 pandemic. In this session data from the thoracic cancers international COVID-19 collaboration (TERAVOLT) registry and from the COVID-19 and cancer consortium registry (CCC-19) were presented [19-21]. The TERAVOLT study focused on thoracic

Table 2 ESMO Magnitude of Clinical Benefit Scale [13]

\begin{tabular}{ll}
\hline Priority level & Patient condition and risk/benefit ratio \\
\hline High priority & $\begin{array}{c}\text { Patient condition is immediately life threatening or clinically unstable, and/or the magnitude of benefit from treatment quali- } \\
\text { fies the interventions as high priority }\end{array}$ \\
Medium priority & $\begin{array}{c}\text { Patient situation is non-critical, but delay beyond 6-8 weeks could potentially affect overall outcome and/or the magnitude of } \\
\text { benefit qualifies for intermediate priority }\end{array}$ \\
Low priority & $\begin{array}{l}\text { Patient's condition is stable enough that services can be delayed for the duration of the COVID-19 pandemic and/or the inter- } \\
\text { vention is no priority based on the magnitude of benefit }\end{array}$ \\
\hline
\end{tabular}


malignancies and recruited 400 patients, mainly from Italy, with either confirmed COVID-19 infection or clinically/radiologically suspected infection [19]. The authors reported a mortality rate of $35 \%$ with a median followup of 33 days. The CCC-19 study reported data on 928 confirmed COVID-19 positive patients, mostly from USA, with various types of cancers [20,21]. The most prevalent malignancies were breast (21\%) and prostate cancer (16\%), while the mortality rate was $13 \%$ with a median followup of 21 days. TERAVOLT data confirmed a high mortality rate among patients with lung cancer who develop COVID-19; in both studies similar independent factors were associated with increased mortality, which included older age, number of comorbidities, poor performance status, active cancer and administration of chemotherapy alone or in combination with other treatments at the time of infection.

Several oncology societies have been quick to develop guidelines on cancer care during COVID-19 pandemic and all these guidelines include a series of recommendations that should be adopted by healthcare workers, patients and their family members or accompanists.

However, there are still many unanswered questions regarding the correct management of cancer patients in the COVID-19 era, including the need to stop certain types of therapies or the risk of impairing the outcome when continuing treatments. Some experts have already emphasized the "distraction effect" of the pandemic, which is represented by the risk of shifting total attention away from standard clinical care to COVID-19 only [22]. In fact it is desirable that physicians even during the pandemic should avoid, if possible, delaying any curative interventions (chemotherapy, radiation, surgery). In any case, as previously reported, ESMO, NICE and French guidelines suggested to use a tiered approach to categorize patients into different priority levels to receive active cancer therapy; Hanna et al. also proposed a useful framework for prioritizing cancer therapy in the pandemic era [23].

In conclusion, during the COVID-19 pandemic the adoption of both proactive management and containment measures, as well as adherence to main available recommendations, can help to protect healthcare workers and patients from possible contamination and to choose the best therapeutic strategy for the patients.

Acknowledgements The authors thank Marina Tartarone for English editing.

\section{Compliance with ethical standards}

Conflict of interest The authors have no conflicts of interest.

\section{References}

1. https://www.who.int/emergencies/diseases/novel-coronaviru s-2019/events-as-they-happen. Accessed 1 May 2020.

2. https://www.who.int/news-room/commentaries/detail/modes-oftransmission-of-virus-causing-covid-19-implications-for-ipcprecaution-recommendations. Accessed 1 May 2020.

3. Verity R, Okell LC, Dorigatti I, et al. Estimates of the severity of coronavirus disease 2019: a model-based analysis. Lancet Infect Dis. 2020;S1473-3099(20):30243-7. https://doi.org/10.1016/ S1473-3099(20)30243-7.

4. Zhang L, Zhu F, Xie L, et al. Clinical characteristics of CIVID19 infected cancer patients: a retrospective case study in three hospitals within Wuhan, China. Ann Oncol. 2020;S09237534(20):36383-93. https://doi.org/10.1016/j.annon c.2020.03.296.

5. Liang W, Guan W, Chen R, et al. Cancer patients in SARSCoV-2 infection: a nationwide analysis in China. Lancet Oncol. 2020;21(3):335-7.

6. Yu J, Ouyang W, Chua MLK, et al. SARS-CoV-2 transmission in patients with cancer at a tertiary care hospital in Wuhan, China. JAMA Oncol. 2020. https://doi.org/10.1001/jamao ncol.2020.0980.

7. Characteristics of COVID-19 patients dying in Italy. https://www. epicentro.iss.it/coronavirus/bollettino/Report-COVID-2019_29_ april_2020. Accessed 1 May 2020.

8. Mehta V, Goel S, Kabarriti R, et al. Case fatality rate of cancer patients with COVID-19 in a New York Hospital system. Cancer Discov. 2020. https://doi.org/10.1158/2159-8290.CD-20-0516.

9. Rischio infettivo da coronavirus COVID 19: indicazioni per l'oncologia. https://www.aiom.it/wp-content/uploa ds/2020/03/20200313_COVID-19_indicazioni_AIOM-CIPOM O-COMU.pdf. Accessed 13 Mar 2020.

10. La gestione delle pazienti con carcinoma mammario durante la pandemia COVID-19 versione 25 Aprile 2020. https://www.aiomit/la-gestione-delle-pazienti-con-carcinoma-mammario-durantela-pandemia-covid-19/. Accessed 1 May 2020.

11. You B, Ravaud A, Canivet A, et al. The official French guidelines to protect patients with cancer against SARS-CoV-2 infection. Lancet Oncol. 2020;S1470-2045(20):30204-7. https://doi. org/10.1016/S1470-2045(20)30204-7.

12. COVID-19 rapid guideline: delivery of systemic anticancer treatments. NICE guideline. https://www.nice.org.uk/guidance/ng161 . Accessed 20 Mar 2020.

13. ESMO Guidelines: Cancer patient management during the COVID-19 pandemic. https://www.esmo.org/guidelines/cance r-patient-management-during-the-covid-19-pandemic. Accessed 1 May 2020.

14. COVID-19 patient care information. https://www.asco.org/ascocoronavirus-information/care-individuals-cancer-during-covid-19. Accessed 1 May 2020.

15. CDC guidance for COVID-19. https://www.cdc.gov/coronaviru s/2019-ncov/hcp/clinical-criteria.html. Accessed 1 May 2020.

16. Guidance on the management of clinical trials during the COVID19 (coronavirus) pandemic. https://ec.europa.eu/health/sites/ health/files/files/eudralex/vol-10/guidanceclinicaltrials_covid 19_en.pdf. Accessed 1 May 2020.

17. Clinical trial conduct during the COVID-19 pandemic. https:// www.fda.gov/drugs/coronavirus-covid-19-drugs/clinical-trial -conduct-during-covid-19-pandemic. Accessed 1 May 2020.

18. NCI statement on clinical trials during COVID-19 pandemic. https://www.cancer.gov/news-events/press-releases/2020/nci-state ment-clinical-trials-during-covid-19. Accessed 1 May 2020.

19. Horn L, Whisenant JG, Torri V, et al. Thoracic cancers international COVID-19 collaboration (TERAVOLT): impact of type of 
cancer therapy and COVID therapy on survival. J Clin Oncol. 2020;38(18):LBA111.

20. Warner JL, Rubinstein S, Grivas P, et al. Clinical impact of COVID-19 on patients with cancer: data from the COVID-19 and cancer consortium (CCC19). J Clin Oncol. 2020;38(18):LBA110.

21. Kuderer NM, Choueiri TK, Shah DP, et al. Clinical impact of COVID-19 on patients with cancer (CCC19): a cohort study. Lancet. 2020;S0140-6736(20):31187-9.

22. Cortiula F, Pettke A, Bartoletti M, et al. Managing COVID-19 in the oncology clinic and avoiding the distraction effect. Ann Oncol. 2020;31(5):553-5.
23. Hanna TP, Evans GA, Booth CM. Cancer, COVID-19 and the precautionary principle: prioritizing treatment during a global pandemic. Nat Rev Clin Oncol. 2020;17(5):268-70.

Publisher's Note Springer Nature remains neutral with regard to jurisdictional claims in published maps and institutional affiliations. 